\title{
W-Band Waveguide-Packaged InP HEMT Reflection Grid Amplifier
}

\author{
Younkyu Chung, Member, IEEE, Chun-Tung Cheung, Michael P. DeLisio, Senior Member, IEEE, and
}

David B. Rutledge, Fellow, IEEE

\begin{abstract}
This letter presents a 79-GHz broadband reflection-type grid amplifier using spatial power combining to combine the power of 64 unit cells. Each unit cell uses a two-stage cascade configuration with InP HEMTs arranged as a differential pair. A broadband orthogonal mode transducer (OMT) separates two orthogonally polarized input and output signals over a 75 to $85 \mathrm{GHz}$ range. In conjunction with the OMT, a mode converter with quadruple-ridged apertures was designed to enhance the field uniformity over the active grid. Measurements show 5-dB small signal gain at $79 \mathrm{GHz}$ and an 800-MHz 3-dB bandwidth. The amplifier generates an output power of $264 \mathrm{~mW}$ with little evidence of saturation.
\end{abstract}

Index Terms-Grid amplifier, mode converter, orthogonal mode transducer (OMT), spatial power combining.

\section{INTRODUCTION}

$\mathbf{S}^{\mathrm{p}}$ PATIAL power combining techniques have shown potential for high power applications at high operating frequencies [1]. This is essentially due to the capability to combine many solid-state devices in free space. Large-scale power combining is difficult using conventional power combining schemes because of inevitable loss through signal lines [2].

Recently, high-performance grid amplifiers using spatial power combining have been extensively investigated and reported [3]-[5]. Grid amplifiers have been realized as both transmission and reflection type. The former has separate input and output signal transmission planes, while those signals coexist in the same plane in the latter [3]. DeLisio et al. demonstrated a 31-GHz two-stage transmission grid amplifier module and over $10 \mathrm{~W}$ output power and 12-dB gain [4]. Cheung et al. presented $82-\mathrm{GHz}$ reflection grid amplifier with $5.5-\mathrm{dB}$ gain, 400-MHz 3-dB bandwidth, and 110-mW saturated output power [5]. While reflection type amplifiers have excellent thermal properties, they often suffer from narrow bandwidths. This is because the input and output are in the same transmission path so that both the input and the output matching structures must be shared.

Manuscript received November 14, 2005; revised February 27, 2006. This work was supported by the Lee Center for Advanced Networking at California Institute of Technology.

Y. Chung was with Information Science and Technology, California Institute of Technology (Caltech), Pasadena, CA 91125 USA and he is now with Freescale Semiconductor Inc., Tempe, AZ 85284 USA (e-mail: ykchung @ieee. org).

C.-T. Cheung and M. P. DeLisio are with Wavestream Corporation, San Dimas, CA 91773 USA.

D. B. Rutledge is with the California Institute of Technology (Caltech), Pasadena, CA 91125 USA (e-mail: rutledge@ caltech.edu).

Digital Object Identifier 10.1109/LMWC.2006.875629

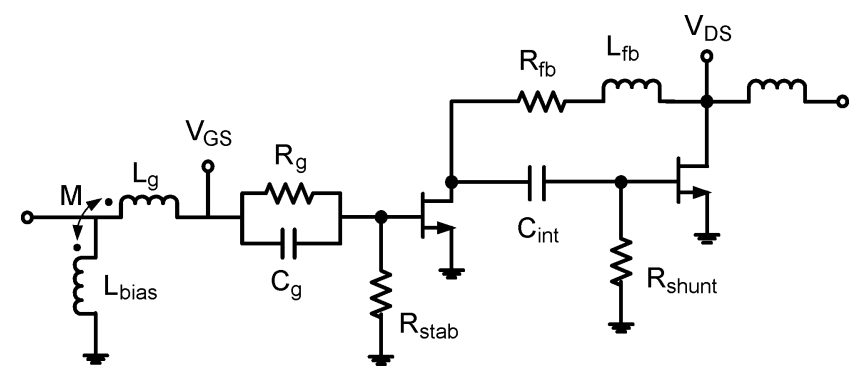

Fig. 1. Simplified RF schematic of bisected circuit of the differentially-driven cascade transistor pair in the unit-cell.

A W-band reflection grid amplifier with 264-mW unsaturated output power and $0.8-\mathrm{GHz} 3-\mathrm{dB}$ bandwidth was developed. A broadband orthogonal mode transducer (OMT) and mode converter with quadruple ridged apertures was employed to improve the frequency bandwidth.

\section{Circuit Design}

\section{A. Two-Stage Cascade Unit Cell}

Due to the relatively small gain of a single-stage unit cell at millimeter-wave frequencies, a two-stage cascade was employed and designed for the unit cell of the grid amplifier. Each unit cell consists of a pair of differentially-driven transistors, gate and drain leads, and bias circuitry. In Fig. 1 the bisected circuit of the differential transistor pair is shown.

The drain bias voltage of the first stage is applied through the feedback resistor $\left(R_{\mathrm{fb}}\right)$. The gate voltage of the second transistor is self-biased by means of the shunt bias resistor $\left(R_{\text {shunt }}\right)$. The interstage matching between the two transistors is essentially accomplished by elements $L_{\mathrm{fb}}, R_{\mathrm{fb}}$, and $C_{\text {int }}$. The $R C$ $\left(R_{\mathrm{g}}\right.$ and $C_{\mathrm{g}}$ ) parallel circuit is used for the input impedancetransformation to $20 \Omega$. The circuit was stabilized by $R_{\mathrm{g}}$ and $R_{\text {stab. }}$

The design of the gate and drain leads connected to the transistor pairs was done by following the modeling procedures described in [6]. The gate peripheries of the first and second stage HEMTs are $80 \mu \mathrm{m}$ and $150 \mu \mathrm{m}$, respectively. An $8 \times 8$ array of the unit cells with a $5.5 \mathrm{~mm} \times 5 \mathrm{~mm}$ total size was used for the final grid chip. The designed grid was fabricated by using Northrop Grumman Space Technology's 0.15- $\mu \mathrm{m}$ InP HEMT process, which is used in [5].

\section{B. Waveguide OMT and Mode Converter}

The passive waveguide in this work is essentially composed of three functional sections illustrated in Fig. 2: an OMT, a mode converter, and an impedance matching section for the active grid 


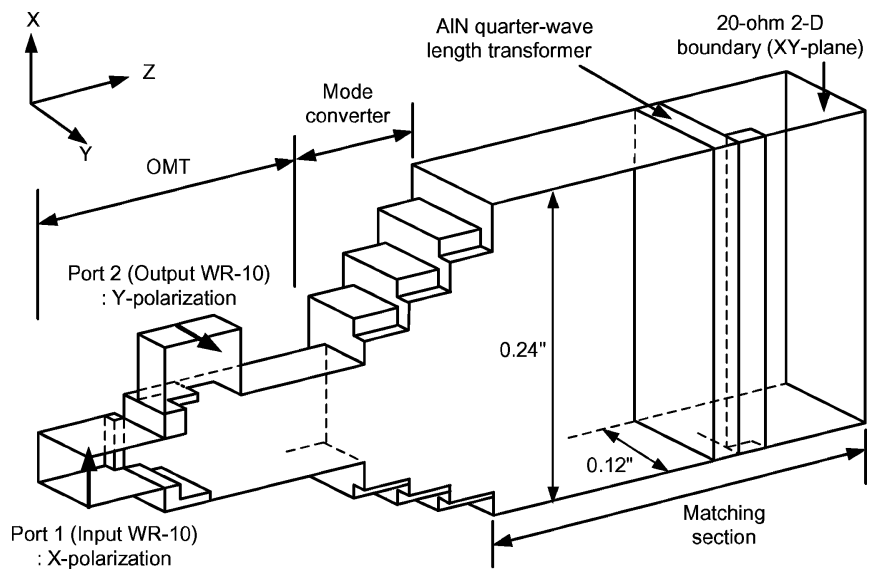

Fig. 2. Illustration of the waveguides in HFSS simulation including the OMT, mode converter, matching parts. This is the symmetrical cross section along the $\mathrm{TE}_{10}$ polarization ( $X Z$-plane).

chip. Ansoft's HFSS full-wave simulator was used for the design of those waveguide parts.

Since both orthogonally polarized input and output signals coexist in the same plane, the OMT is very important. As a threeport device, the OMT separates two orthogonally polarized signals into two standard waveguide ports, while providing a low cross-coupling, a high port-isolation, a low transmission loss, and low input and output return losses. The design specifications of the waveguide section with the OMT and mode converter for the input/output matching and port-isolation to be better than $-10 \mathrm{~dB}$ and $-30 \mathrm{~dB}$, respectively, over the $75-85 \mathrm{GHz}$ frequency range.

Many unit cells are incorporated in the grid amplifier so that the size of the array is larger than that of single-mode waveguides. This requires a mode converter which transforms a standard waveguide to an over-moded one while avoiding any excitation of undesired modes. The third port of the OMT, as shown in Fig. 2, was expanded to the over-moded waveguide through the mode converter. The main role of the mode converter is to obtain selected modes $\left(\mathrm{TE}_{10}\right.$ and $\mathrm{TE}_{30}$ ) with proper magnitude and phase so that the field flatness over the aperture of the active grid can be maximized. Furthermore the mode converter is required to suppress undesired higher order modes such as $\mathrm{TE}_{21}$ and $\mathrm{TM}_{21}$ and to provide low input and output return loss. In this work, three compact intermediate waveguide steps were used to expand the aperture size to 0.24 in $\times 0.24$ in. Symmetrical quadruple ridged-type waveguide sections were used for enhancing frequency bandwidth. The suppression goal of the OMT and mode converter was set to $-20 \mathrm{~dB}$ below for the $\mathrm{TE}_{21}$ and $\mathrm{TM}_{21}$ modes at target frequencies.

The other section of the waveguide is for impedance matching. The grid chip was modeled as a $20-\Omega$ 2-D impedance boundary on top of the InP substrate in the HFSS simulation. The 10-mil-thick Aluminum Nitride (AIN) is used as a heat spreader as well as a tuning element; it is placed under the gird against the back short. In addition to the AlN substrate, the impedance matching was accomplished by placing an additional AlN quarter-wave length transformer and an iris as shown in Fig. 2.

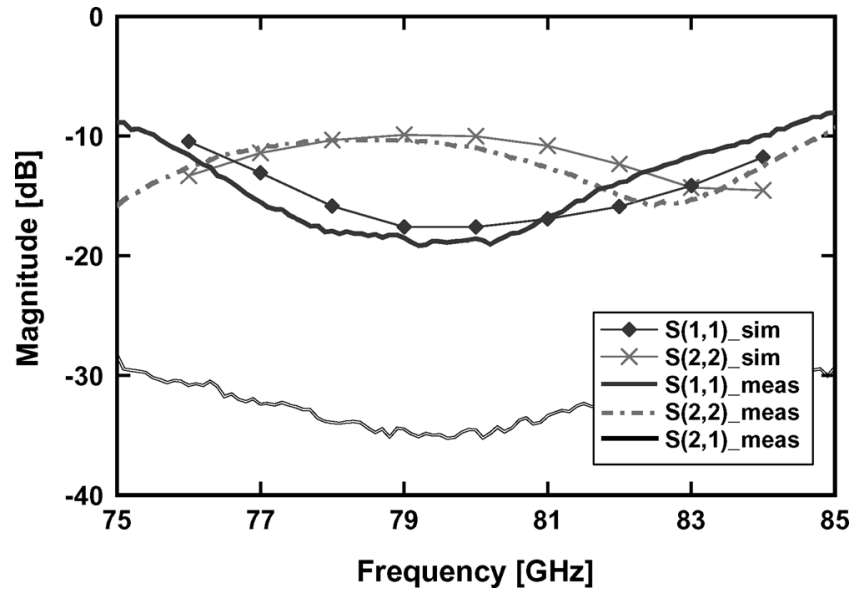

Fig. 3. Measured $\left(S_{\text {meas }}\right)$ and HFSS-simulated $\left(S_{\text {sim }}\right) S$-parameters of the OMT in conjunction with mode converter over 75 to $85 \mathrm{GHz}$.

\section{MEASUREMENT RESUltS}

The designed waveguides described in Section II were fabricated out of brass and gold plated to reduce metallic loss. Due to the complexity of the OMT, it was split into two blocks along the center of $\mathrm{TE}_{10}$ input polarization and combined together. Each waveguide step of the mode converter was built separately and all fabricated sections were stacked together. The measured $S$-parameters of the implemented OMT with the mode converter are shown in Fig. 3. The measured return loss for the input and output ports and the port-isolation agree well with the simulation results. The transmission loss between the input/output ports and the end of the mode converter is less than $0.5 \mathrm{~dB}$ over the design frequency.

To assemble active grid chip, a 10-mil-thick AlN heat spreader was first mounted on top of the brass back short. Then, the chip was mounted on top of the AlN substrate by means of a non-conductive epoxy with a high thermal conductivity. Gold wires were bonded to provide de bias. For the drain (second stage) and gate (first stage) bias circuits, microstrip low-pass filters with nulls around $79 \mathrm{GHz}$ are used to prevent RF signal leakage to dc supply ports. The bias circuitry was printed on 10-mil-thick RT/Duroid substrate with a dielectric constant of 2.2. Fig. 4(a) shows a photograph of the mounted grid amplifier chip with bias circuitry. Then, both the mounted active grid and passive waveguide sections including the OMT, mode converter, and matching section were combined together. The assembled grid amplifier with an air-cooled heat sink is shown in Fig. 4(b).

The small-signal performance of the grid amplifier was tested first. The measured $S$-parameters over 77 to $81 \mathrm{GHz}$ are shown in Fig. 5. The peak gain of about $5 \mathrm{~dB}$ at $78.8 \mathrm{GHz}$ was achieved with the $V_{\mathrm{DS}}=1.8 \mathrm{~V}$ and $V_{\mathrm{GS}}=-0.3 \mathrm{~V}$. As shown in Fig. 5, a 3-dB-gain bandwidth of $800 \mathrm{MHz}$ was obtained. Compared to the 400-MHz bandwidth of [5], the bandwidth of the implemented grid was significantly expanded due to the OMT, mode converter, and matching. The input and output return loss below $-10 \mathrm{~dB}$ was achieved over a frequency range from 78.5 to $79.5 \mathrm{GHz}$. Note that there was no oscillation observed in 


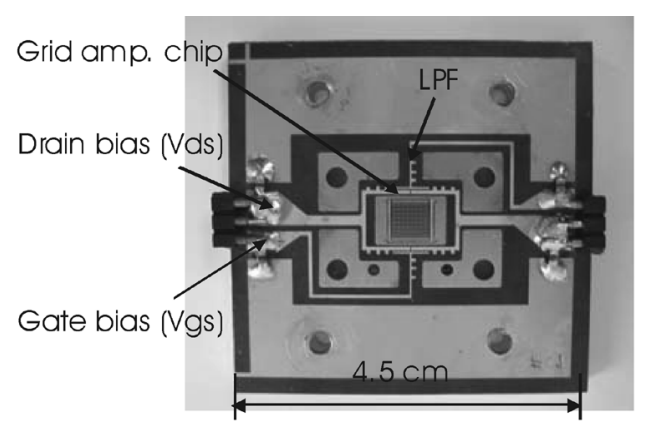

(a)

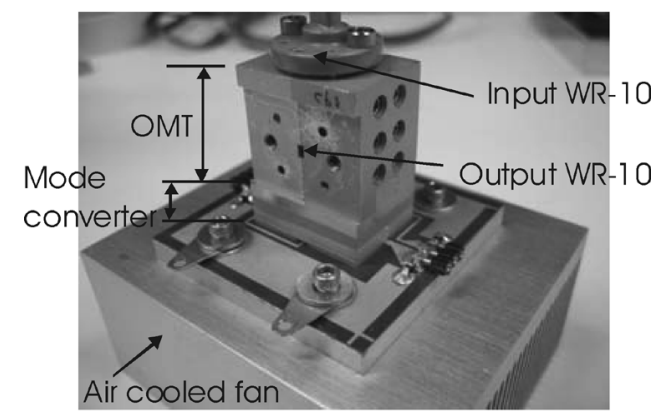

(b)

Fig. 4. (a) Photograph of mounted grid chip. (b) Photograph of assembled grid amplifier.

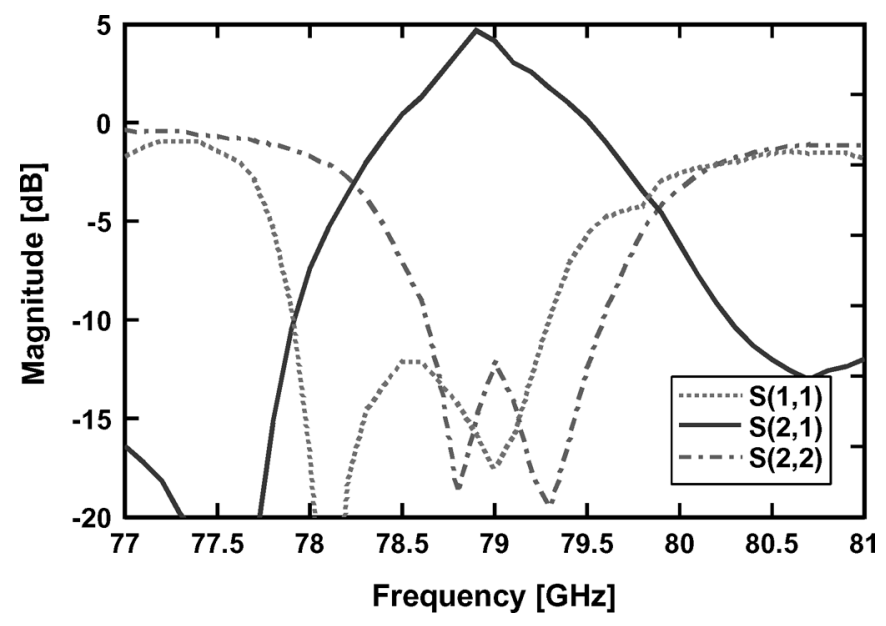

Fig. 5. Measured small signal $S$-parameters of the assembled grid amplifier.

the measurement because the OMT suppresses the coupling between the input and output signals.

A driver module was used for the input source of the grid amplifier in large-signal measurement. The maximum output power of the driver at $79 \mathrm{GHz}$ is limited to $20 \mathrm{dBm}$ in the measurement, which was not sufficient to saturate our amplifier. In Fig. 6, the measured output power and gain with respect to the applied input power is shown. Note that the $V_{D S}$ was set to $2.2 \mathrm{~V}$ and dc drain current was $9.1 \mathrm{~A}$ in the measurement. The unsaturated maximum output power of $24.2 \mathrm{dBm}(264 \mathrm{~mW})$ with a maximum gain of $5.2 \mathrm{~dB}$. Note that the ideal power density per

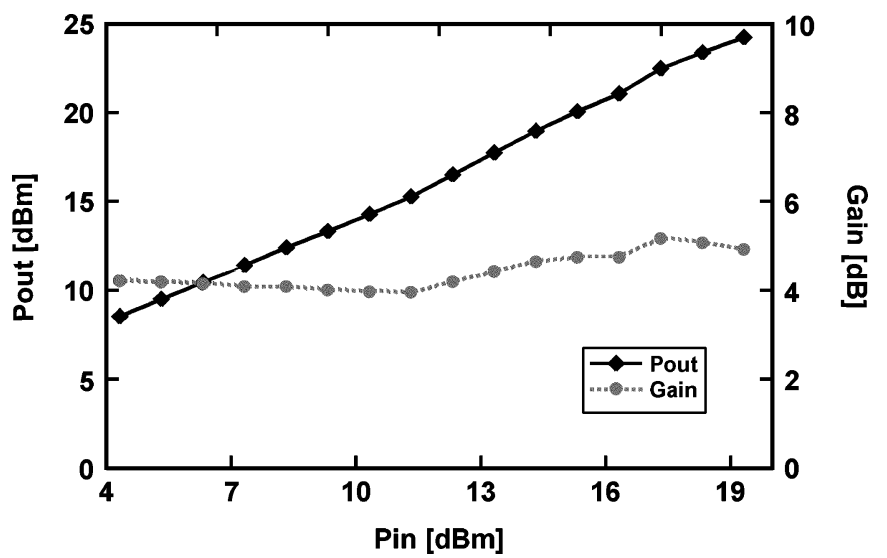

Fig. 6. Measured large-signal performance of the grid amplifier with respect to the input power.

gate periphery of the HEMT is $300 \mathrm{~mW} / \mathrm{mm}$ [5]. As clearly seen in Fig. 6, the implemented grid amplifier does not show output power-saturation behavior up to the maximum attainable input power level. The output power can be increased with a higher power driver. The gain variation may be caused by a weak nonlinear behavior of the unit cells, resulting in the gain expansion of the gird amplifier at the input power level higher than $12 \mathrm{dBm}$.

\section{CONCLUSION}

A W-band reflection grid amplifier with an $800-\mathrm{MHz} 3-\mathrm{dB}-$ gain bandwidth is demonstrated. A $260-\mathrm{mW}$ unsaturated output power with 5-dB small-signal gain was achieved from the implemented grid amplifier.

\section{ACKNOWLEDGMENT}

The authors would like to thank Dr. J. Kuno and J. Ma, Quinstar technology Inc., for their generous help with the large-signal measurement, R. Tsai, Northrop Grumman, for fabrication of the grid chips, and Dr. S. Weinreb, Jet Propulsion Laboratory, for his helpful discussions.

\section{REFERENCES}

[1] R. A. York and Z. B. Popovic, Active and Quasi-Optical Arrays for Solid-State Power Combining. New York: Wiley, 1997.

[2] R. A. York, "Some consideration of optimal efficiency and low noise in large power combiners," IEEE Trans. Microw. Theory Tech., vol. 49, no. 8, pp. 1477-1482, Aug. 2001.

[3] C.-T. Cheung, R. Tsai, R. Kagiwada, and D. B. Rutledge, "V-band transmission and reflection Grid amplifier packaged in waveguide," in IEEE MTT-S Int. Dig., 2003, vol. 1, pp. 1863-1866.

[4] M. P. DeLisio, B. Deckman, C.-T. Cheung, S. C. Martin, D. P. Nakhla, E. E. Hartmann, C. J. Rollison, J. B. Pacetti, and J. Rosenberg, "A Ka-band Grid amplifier module with over 10 Watts output power," in IEEE MTT-S Int. Dig., 2004, vol. 1, pp. 83-86.

[5] C.-T. Cheung, M. P. DeLisio, J. Rosenberg, R. Tasi, R. Kagiwada, and D. B. Rutledge, "A single chip two-stage W-band Grid amplifier," in IEEE MTT-S Int. Symp. Dig., 2004, vol. 1, pp. 79-82.

[6] P. Preventza, B. Eickman, E. Sovero, M. P. DeLisio, J. J. Rosenberg, and D. B. Rutledge, "Modeling of quasi-optical arrays," in IEEE MTT-S Int. Dig., 1999, vol. 2, pp. 563-566. 